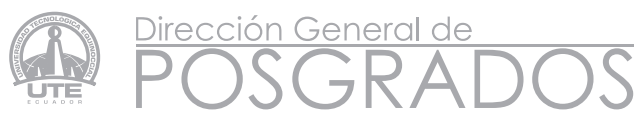

\section{INVENTARIO DEL CICLO DE VIDA DEL PROCESO DE PRODUCCIÓN DE BAMBÚ ROLLIZO TRATADO DE LA ESPECIE DENDROCALAMUS ASPER EN EL NOROCCIDENTE DE PICHINCHA}

${ }^{1}$ Andrea Jaramillo Benavides, ${ }^{2}$ Lisiane Ilha Librelotto, ${ }^{3}$ Myrian Larco Benítez

\author{
'Universidade Federal de Santa Catarina, Florianópolis - Brasil, andresalome@gmail.com \\ 2Universidade Federal de Santa Catarina, Florianópolis - Brasil, lisiane.librelotto@gmail.com \\ 3Universidad Tecnológica Equinoccial, Quito - Ecuador, myrian.larco@ute.edu.ec
}

Recepción / Received: 06,10, 2015

Aceptación / Accepted: 22,12, 2015

Publicado / Published: 30,12, 2015

\section{Resumen:}

El Análisis de Ciclo de Vida de materiales de construcción locales, contribuye a la elaboración de bases de datos, donde se muestre y cuantifique el impacto ambiental de los procesos necesarios para la fabricación, uso y descarte de cada material; siendo una herramienta de apoyo en la toma de decisiones de diseño enfocadas hacia una arquitectura y procesos constructivos más sostenibles. El presente trabajo muestra un estudio de Inventario de Ciclo de Vida realizado en una empresa del noroccidente de Pichincha sobre el proceso productivo de tallos de bambú Dendrocalamus asper tratado, comercializados principalmente para su aplicación como estructura en edificaciones. El método fue desarrollado conforme los procedimientos indicados en la norma ISO 14042. Los resultados indican los factores de mayor impacto ambiental durante el proceso, en este caso el combustible usado para el transporte del producto.

\section{Palabras clave:}

Inventario de Ciclo de Vida, bambú, Dendrocalamus asper.

\begin{abstract}
:
The Life Cycle Assessment of local building materials contributes to the development of databases that display and quantify the environmental impact of manufacture processes, use and disposal of each material. It is a support tool to make design decisions towards more sustainable architecture and construction processes. This paper presents a case of Life Cycle Inventory developed in a company situated in northwestern Pichincha; it is about the production process of bamboo Dendrocalamus asper treated stalks, which are used primarily as building structure. The method was developed in accordance with the ISO14042 procedures. The results indicate the environmental impact factors during the process; in this case the biggest impact comes from the fuel used during the transportation.
\end{abstract}

\section{Keywords:}

Life Cycle Assessment, bamboo, Dendrocalamus asper. 


\section{INTRODUCCIÓN}

El auge actual de la búsqueda de la sostenibilidad en la arquitectura abre varios ámbitos para la investigación en el área, entre ellos la búsqueda de materiales de construcción que, además de tener buenas características físicas y mecáni cas, sean más amigables con el medio. Una de las herramientas que permite evaluar y cuantificar el impacto ambiental de los materiales, componentes y sistemas constructivos es la Evaluación o Análisis del Ciclo de Vida (ACV).

Suppen y van Hoof (2005) afirman que la metodología del ACV tiene una estructura que permite calcular cuantitativamente medidas para después analizar integralmente los perfiles ambientales de los productos. De esta manera se pueden realizar comparaciones entre las características ambientales de cada material para saber cuál elegir a la hora de realizar un proyecto arquitectónico sostenible.

El ACV consiste en realizar un inventario de entradas y salidas (de materia y energía) del sistema estudiado; en este caso del proceso de fabricación de un material - desde la extracción de materias primas hasta el descarte o reutilización. Es una herramienta científica usada para levantar, cuantificar y comparar esta información entre materiales de construcción que pueden tener la misma función en un edificio pero diferentes impactos ambientales durante su ciclo de vida.

En el ACV son determinados valores para cada etapa de vida de un producto: producción de materia prima, transporte, manufactura, distribución, descarte- reciclaje (Thompson, 2005). Es por esto que sirve además para identificar las fases del proceso de producción o fabricación de los materiales que podrían ser optimizadas para mejorar el perfil ambiental del producto.

Por ejemplo, un trabajo de ACV, realizado por Hernández et al. (2012) que estudió el proceso de extracción de bambú Guadua rollizo en el Eje Cafetero en Colombia, mostró que los impactos ambientales más relevantes se relacionaron con el consumo de combustible usados para aserrar y transportar el material. Otro estudio desarrollado con bambú rollizo de la especie Mosó en Asia por Lugt et al (2009) indica que casi todos los costos ambientales de ese bambú son ocasionados por el transporte marítimo de esos tallos hacia Europa (donde posteriormente son industrializados); lo que evidenció la importancia de potenciar el consumo y transformación local de las materias primas.

Internacionalmente las normas ISO 14000 tratan temas de calidad y gestión ambiental. En Ecuador se adoptaron estas normas y dentro de las normas INEN-ISO 14040 se trata el tema específico del ACV. Esta norma indica que un estudio de ACV consta de cuatro fases:

a) Definición del objetivo y campo de aplicación.

b) Análisis de inventario (ICV)

c) Evaluación de impacto ambiental (EICV)

d) Interpretación

En este trabajo se desarrolló una parte del inventario necesario para la realización del ACV del bambú rollizo tratado, ya que no se analizan las etapas de aplicación, mantenimiento y descarte (o reutilización) del material.

El presente artículo muestra la metodología usada y los resultados obtenidos en este caso específico.

El bambú analizado en este estudio es de la especie Dendrocalamus asper (conocido comúnmente como bambú gigante) que fue introducida en Ecuador a fines del siglo XX y ha tenido una buena adaptación al medio. En el noroccidente de Pichincha y zonas limítrofes de la provincia existen alrededor de 1200 ha. establecidas con fines comerciales, distribuidas entre 57 productores. (ALFARO, 2010).

Los culmos de este bambú pueden ser usados enteros en estructuras o laminados para fabricar pisos, tableros, vigas, muebles y otros productos. Este trabajo analiza únicamente el bambú rollizo tratado, dejando el análisis de otros subproductos para investigaciones posteriores.

Los datos usados para los cálculos fueron recolectados de una empresa proveedora de bambú del noroccidente, localizada a $128 \mathrm{Km}$ de Quito; que fue seleccionada porque realiza el proceso completo desde la silvicultura, el tratamiento de preservación y secado de los culmos de bambú (usando métodos que son comunes entre los productores de la zona) y finalmente la comercialización. 


\section{OBJETIVO Y CAMPO DE APLICACIÓN}

Este estudio tiene como objetivo obtener información sobre el flujo de materia y energía durante la producción de bambú rollizo tratado; desde la etapa de manejo de la plantación, pasando por la etapa de colecta, transporte y tratamiento; que concluye con la comercialización de los culmos tratados a los compradores.

Los resultados servirán de base para el análisis completo de ciclo de vida del bambú y posteriormente pueden ser insumo para realizar los ACV de otros productos derivados del mismo o de componentes constructivos que lo contengan.

La unidad funcional de referencia adoptada para realizar las medidas de Inventario del Ciclo de Vida (entrada y salida de materia y energía) de las etapas estudiadas en este trabajo es un culmo de bambú tratado de $6 \mathrm{~m}$ de longitud listo para su aplicación.

\section{ANÁLISIS DE INVENTARIO (ICV)}

Es la fase del ACV que incluye la recopilación y cuantificación de entradas y salidas para un determinado producto a lo largo de su ciclo de vida. Las entradas corresponden a la materia o energía que ingresa durante el proceso en relación a la unidad adoptada, mientras las salidas corresponden a la materia o energía que deja el proceso en relación a la misma unidad. (ISO 14040:2006).

Para garantizar la fiabilidad y consistencia de la información en esta etapa, se hizo una transformación de unidades de todos los datos levantados, trayéndolos hasta la unidad funcional definida.

Características de los culmos de bambú.- La empresa estudiada ofrece culmos de bambú de la especie Dendrocalamus asper, de $6 \mathrm{~m}$ de largo (unidad funcional adoptada), su diámetro varía entre $12-15 \mathrm{~cm}$, la distancia media entre nudos es de aproximadamente $40 \mathrm{~cm}$, el espesor de la pared puede ser entre $8-35 \mathrm{~mm}$. (E. Briones, comunicación personal, agosto 28 2015). El tratamiento preservante se realiza por inmersión en una solución de bórax, ácido bórico y agua. Los culmos posteriormente pasan por una etapa de secado al aire libre y en sombra, solo entonces están listos para su comercialización.
En la figura 1 se muestra el proceso productivo de los culmos de bambú tratado. La línea entrecortada indica los límites de las etapas evaluadas en este inventario.

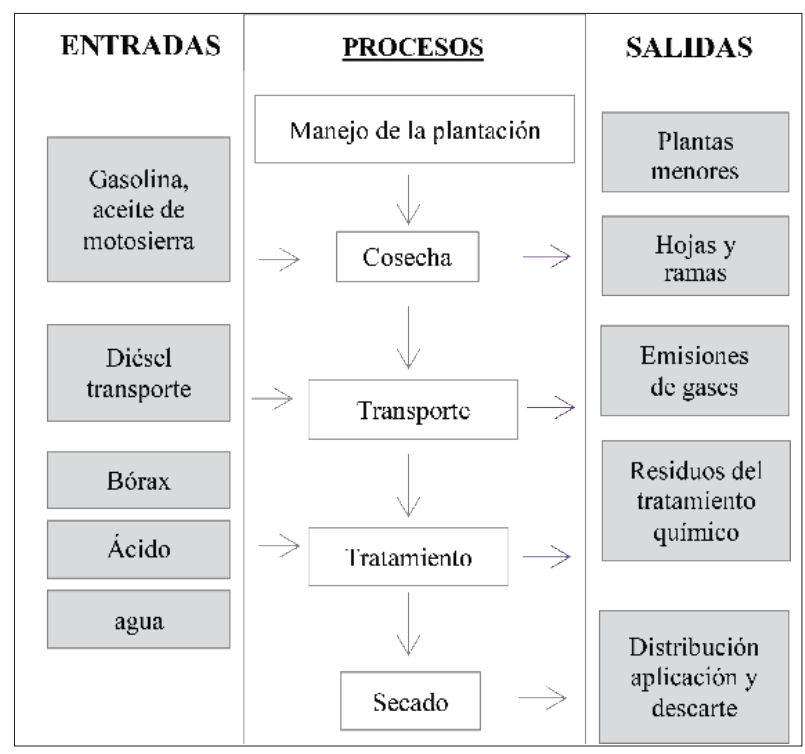

Figura 1. Proceso de producción de bambú rollizo tratado. Fuente: elaboración propia

\section{Manejo de la plantación.-}

Se limitó el proceso de análisis para iniciar desde el manejo del bambusal y no desde la siembra porque las plantaciones ya existen y, en el caso del bambú, no es necesario sembrar más plantas para obtener nuevos culmos, pues nuevos rizomas surgen de los rizomas existentes dando origen a los brotes. A las plantaciones de bambú se las deja crecer en la zona "naturalmente" sin realizar inversiones en fertilizantes (estos son usados únicamente para los dos primeros años cuando las plántulas son nuevas), en la zona no se usan mecanismos de riego adicionales, las plantaciones crecen únicamente con la humedad y precipitaciones naturales, dando una producción estacionaria de nuevos brotes que aparecen al inicio de la época de lluvias.

Los tallos de bambú son marcados en las macollas con cintas de colores dependiendo del año de nacimiento como se muestra en la figura 2. El mantenimiento de las macollas se realiza manualmente con machetes o moto guadañas y consiste principalmente en evitar el crecimiento de plantas menores alrededor que puedan dificultar la posterior extracción de los culmos. Los residuos de estas plantas que quedan tras la limpieza permanecen en el terreno como parte de la capa vegetal de protección, por lo tanto no son cuantificados como salida de material para este inventario. 


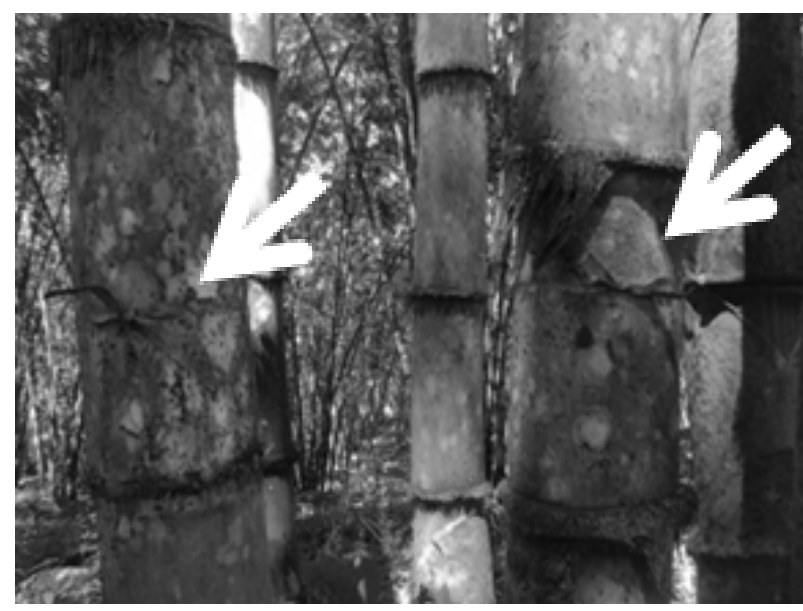

Figura 2. Bambús marcados con cintas en la plantación. Fuente: fotografía propia

El tiempo de uso de la moto guadaña para cada macolla, la edad de colecta del bambú y el rendimiento del combustible/hora para esta herramienta fueron los datos considerados para estimar el consumo de $\mathrm{g}$ asolina y aceite durante esta fase.

Los datos de emisiones debido a la quema de gasolina y aceite se calcularon en base al consumo total en litros y a datos de emisión de contaminantes por litro de combustible quemado. Finalmente se hizo una relación de todos esos datos para la unidad adoptada (un culmo de bambú de $6 \mathrm{~m}$ de largo). La tabla 1 indica los datos base que fueron tomados en cuenta.

Tabla 1. Datos utilizados para cálculos de emisiones en las etapas de manejo de plantación y cosecha

\begin{tabular}{|c|c|c|c|}
\hline \multicolumn{2}{|c|}{ Datos colectados directo de la fuente } & \multicolumn{2}{|c|}{$\begin{array}{l}\text { Datos de emisiones tomados de } \\
\text { otras fuentes }\end{array}$} \\
\hline $\begin{array}{l}\text { Tipo de herramienta } \\
\text { usada }\end{array}$ & $\begin{array}{l}\text { Motosienra y } \\
\text { moto guadaña }\end{array}$ & \multirow{5}{*}{$\begin{array}{l}\text { Emisiones } \\
\text { gaseosas de } \\
\text { la gasolina } \\
\text { y el aceite }\end{array}$} & \multirow{5}{*}{$\begin{array}{c}\mathrm{CO}_{2} 1,5 \mathrm{~kg} / \text { litro } \\
\text { (IEA, 2014) }\end{array}$} \\
\hline Tipo de combustible & $\begin{array}{l}\text { Gasolina y } \\
\text { aceite }(50: 1)\end{array}$ & & \\
\hline $\begin{array}{l}\text { Tiempo que demora } \\
\text { cortar y desramar un } \\
\text { tallo de bambú del } \\
\text { cogollo }\end{array}$ & $10 \mathrm{~min}$ & & \\
\hline $\begin{array}{l}\text { Número de tallos de } \\
\text { bambú por cogollo }\end{array}$ & 200 & & \\
\hline $\begin{array}{l}\text { Rendimiento } \\
\text { combustible }\end{array}$ & 1 litro/hora & & \\
\hline
\end{tabular}

Resumen de la etapa: consumo de gasolina y aceite (entradas); emisión de gases por la quema de gasolina y aceite (salidas).

\section{Cosecha de culmos.-}

La cosecha se realiza manualmente, con la ayuda de una motosierra, en el mismo lugar se cortan los culmos del tamaño adecuado para el transporte (ver figura 3). Se limpian los tallos quitando las ramas y con una varilla afilada se perforan todos los nudos internospara extraer el agua y facilitar el transporte.

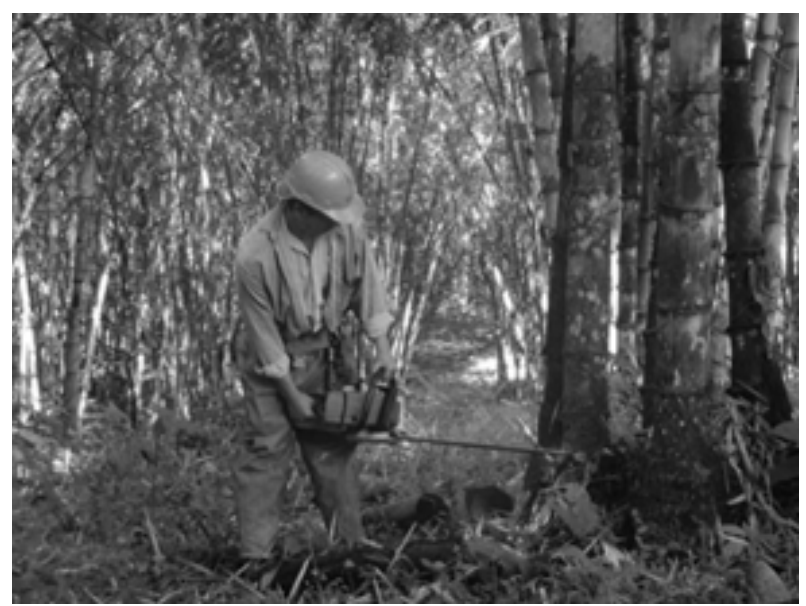

Figura 3. Cosecha de Dendrocalamus asper.

Fuente: fotografía propia

De cada tallo de bambú se obtienen 3 culmos de $6 \mathrm{~m}$ de largo, sobrando la parte basal de aproximadamente $2 \mathrm{~m}$ (que se usa para hacer latillas), también la parte más alta de unos $4 \mathrm{~m}$ de largo (que se usa como puntales en otras plantaciones como banano y cacao) y las hojas se quedan en el terreno (siendo aprovechadas como materia orgánica para el suelo).

Posteriormente los culmos son cargados, sin uso de maquinaria, hasta el camión que los llevará al sitio de tratamiento.

Los datos considerados para calcular las emisiones en esta fase fueron: el tiempo de uso de la motosierra para cortar y desramar un tallo, el rendimiento del combustible/hora para esta herramienta.

Las emisiones por quema de gasolina y aceite se calcularon de acuerdo al consumo total en litros y a datos de emisión de contaminantes por litro de combustible quemado. Finalmente se hizo una relación de todos esos datos para la unidad adoptada (culmo de $6 \mathrm{~m}$ de largo).

Resumen de la etapa: consumo de gasolina y aceite (entradas); bambú basal que será latillado, ramas y hojas, emisión de gases por la quema de gasolina y aceite (salidas).

\section{Transporte.-}

Los culmos de bambú son trasladados en camiones al lugar donde se realizará el tratamiento preservativo y el secado. Al llegar al sitio se verifica que estén limpios y que todos los nudos internos estén perforados, ver figura 4. 
Para el cálculo de consumo de diésel se realizó un promedio del rendimiento combustible/ distancia media de las plantaciones hacia el sitio de tratamiento. Luego se estimó las emisiones por quema de diesel relacionando los litros quemados con la cantidad de gases contaminantes liberados, con equivalencias tomadas de Braun et al(2003).

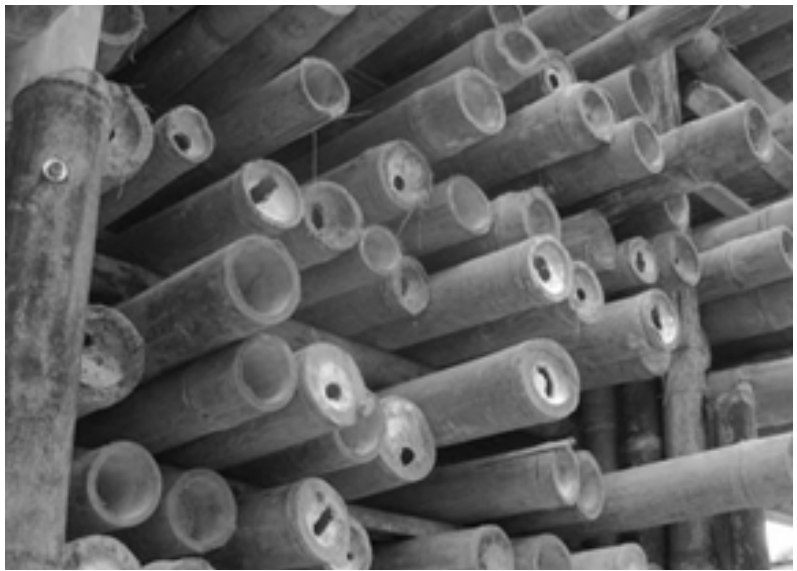

Figura 4. Culmos con nudos internos perforados. Fuente: fotografía propia

Para el cálculo de consumo de diésel se realizó un promedio del rendimiento combustible/ distancia media de las plantaciones hacia el sitio de tratamiento. Luego se estimó las emisiones por quema de diesel relacionando los litros quemados con la cantidad de gases contaminantes liberados, con equivalencias tomadas de valores presentados por Braun et al (2003). En la tabla 2 se muestran estos datos.

\begin{tabular}{lcr}
\multicolumn{2}{l}{ Datos colectados directo de la fuente } & \\
\hline $\begin{array}{l}\text { Tipo de transporte } \\
\text { utilizado }\end{array}$ & Camión & $\begin{array}{r}\text { CO 3-30 g/K } \\
\text { HC 0.5-10 g/l } \\
\text { NOpo de combustible } 0.5-5 \mathrm{~g} / \mathrm{l}\end{array}$ \\
$\begin{array}{l}\text { Tistancia media } \\
\text { recorrida }\end{array}$ & Diésel & SOx 0.5-5 g/l \\
$\begin{array}{l}\text { Número de tallos de } \\
\text { bambú por viaje }\end{array}$ & 200 de $6 \mathrm{~m}$ de \\
$\begin{array}{l}\text { El camión retorna } \\
\text { vacío a la plantación }\end{array}$ & largo \\
$\begin{array}{l}\text { Rendimiento } \\
\text { combustible }\end{array}$ & Sí & Diésel \\
\hline
\end{tabular}

Resumen de la etapa: consumo de diésel (entradas); emisión de gases por la quema de combustible (salidas).

\section{Tratamiento.-}

Se realiza en grupos de 200 unidades, bambús se sumergen en una piscina de $58,5 \mathrm{~m} 3$ con bórax y ácido bórico al 10\% en agua. Luego se secan en posición vertical antes del almacenamiento y secado.

La solución sobrante se almacena en tanques junto a la piscina para ser reutilizada en futuros tratamientos como muestra la figura 5. Se prepara más solución preservante conforme sea necesario.

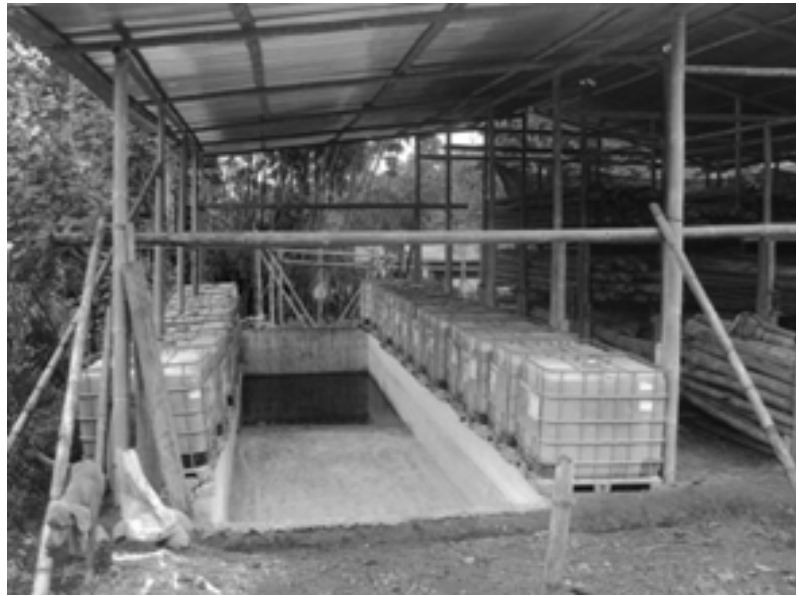

Figura 5. Piscina para tratamiento por inmersión del bambú.

Fuente: fotografía propia\}

Se tomó en cuenta en cuenta que de los 25 tanques de solución, solo 22 vuelven a llenarse después de la inmersión, también el punto de saturación de la fibra del bambú.

Resumen de la etapa: consumo de agua, ácido bórico y bórax (entradas); se consideró que ya que el tratamiento químico es reutilizado, el residuo es despreciable (salidas).

\section{Secado.-}

Este proceso se realiza al natural, sin ayuda de equipamientos: los bambús se apilan horizontalmente, bajo techo, evitando su contacto con el suelo.

Resumen de la etapa: en esta etapa no se registran entradas, la única salida es la evaporación del exceso de humedad en los culmos hasta que alcanzan el punto de equilibrio higroscópico.

En la figura 6 se puede observar el diagrama de flujos ampliado de todo el proceso. 


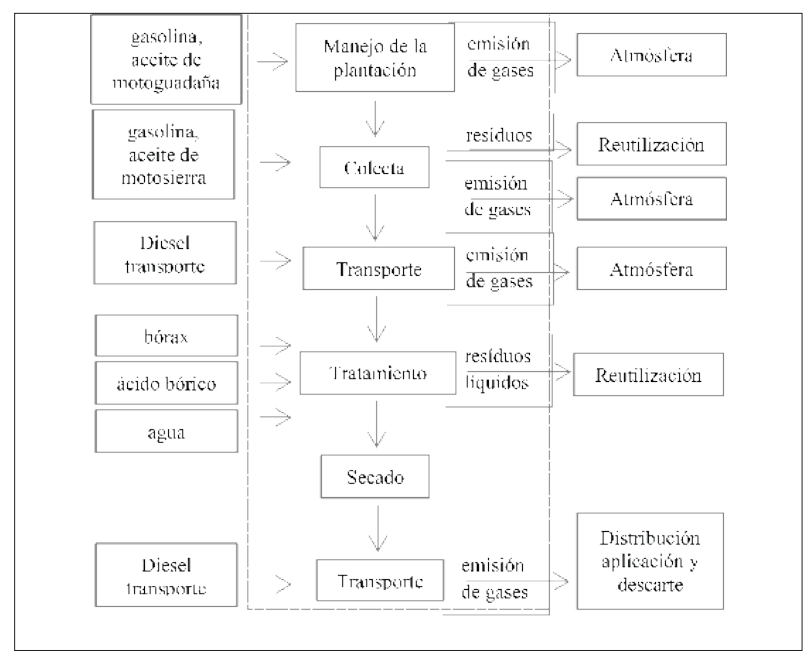

Figura 6. Flujograma de la producción de bambú rollizo tratado

\section{RESULTADOSY DISCUSIÓN}

Los resultados de cada uno de los cálculos realizados sobre entradas y salidas del proceso extrapolados a la unidad funcional se encuentran en la tabla 3.

Tabla 3. Inventario de la producción de un tallo de bambú tratado de $6 \mathrm{~m}$ de longitud. Fuente: Elaboración propia

\begin{tabular}{|c|c|c|c|}
\hline \multicolumn{2}{|c|}{ ENTRADAS } & \multicolumn{2}{|c|}{ SALIDAS } \\
\hline Insumos & $\begin{array}{c}\text { Cantidad } \\
\text { pieza rolliza } \\
\text { de Óm de } \\
\text { largo }\end{array}$ & Item & $\begin{array}{c}\text { Cantidad /pieza } \\
\text { rolliza de } 6 \mathrm{~m} \\
\text { de largo }\end{array}$ \\
\hline gasolina & $\begin{array}{c}0,067 \mathrm{lt} \circ \\
0,05 \mathrm{Kg}\end{array}$ & $\mathrm{CO} 2$ & $0,148 \mathrm{Kg}$ \\
\hline aceite & $\begin{array}{c}0,001 \mathrm{lt} \mathrm{o} \\
0,003 \mathrm{Kg}\end{array}$ & $\mathrm{CO}$ & $0,00768 \mathrm{Kg}$ \\
\hline diésel & $\begin{array}{c}21,66 \text { lt o } \\
0,48 \mathrm{Kg}\end{array}$ & $\mathrm{HC}$ & $0,00264 \mathrm{Kg}$ \\
\hline bórax & $0,0034 \mathrm{lt}$ & NOx & $0,00144 \mathrm{Kg}$ \\
\hline $\begin{array}{l}\text { ácido } \\
\text { bórico }\end{array}$ & $0,0034 \mathrm{lt}$ & $\mathrm{SO} x$ & $0,00144 \mathrm{Kg}$ \\
\hline \multirow[t]{6}{*}{ agua } & $0,06 \mathrm{lt}$ & Partículas & $0,0024 \mathrm{Kg}$ \\
\hline & & bambú tratado & 1 unidad \\
\hline & & $\begin{array}{l}\text { residuo tallo } \\
\text { parte superior } \\
\text { bambú } \\
\text { (reutilizado) }\end{array}$ & $1,33 \mathrm{~m}$ \\
\hline & & $\begin{array}{l}\text { residuo tallo } \\
\text { parte basal } \\
\text { bambú (sale } \\
\text { para latillar) }\end{array}$ & $0,65 \mathrm{~m}$ \\
\hline & & $\begin{array}{l}\text { Solución } \\
\text { química }\end{array}$ & \\
\hline & & $\begin{array}{l}\text { preservante } \\
\text { (siempre se } \\
\text { reutiliza) }\end{array}$ & $110 \mathrm{lt}$ \\
\hline
\end{tabular}

Las mediciones indicadas se realizaron únicamente hasta la fase de secado, cuando el producto está almacenado y listo para su distribución.

Los residuos que son reutilizados fueron cuantificados igualmente para tener una referencia, sin embargo su impacto ambiental es mínimo.

Sería posible posteriormente dar seguimiento al ciclo de vida por ejemplo de la parte del tallo que será usada para hacer latillas, ya es otro proceso que incluye otras variables y podría ser cuantificado.

Debido a la dificultad de medición no fueron cuantificadas las emisiones de vapor de agua que puede salir durante el proceso de secado.

La figura 7 presenta el flujograma del inventario de ciclo de vida con entradas y salidas cuantificadas del proceso de producción de un tallo de bambú rollizo tratado de $6 \mathrm{~m}$ de longitud.

Al final de la figura se puede ver que luego de la fase de secado vendría el transporte, en este caso con línea entrecortada se indican los valores de un ejemplo suponiendo que el material sea transportado hasta Quito en el mismo camión operado a diesel que es usado para sacar el bambú de la plantación.

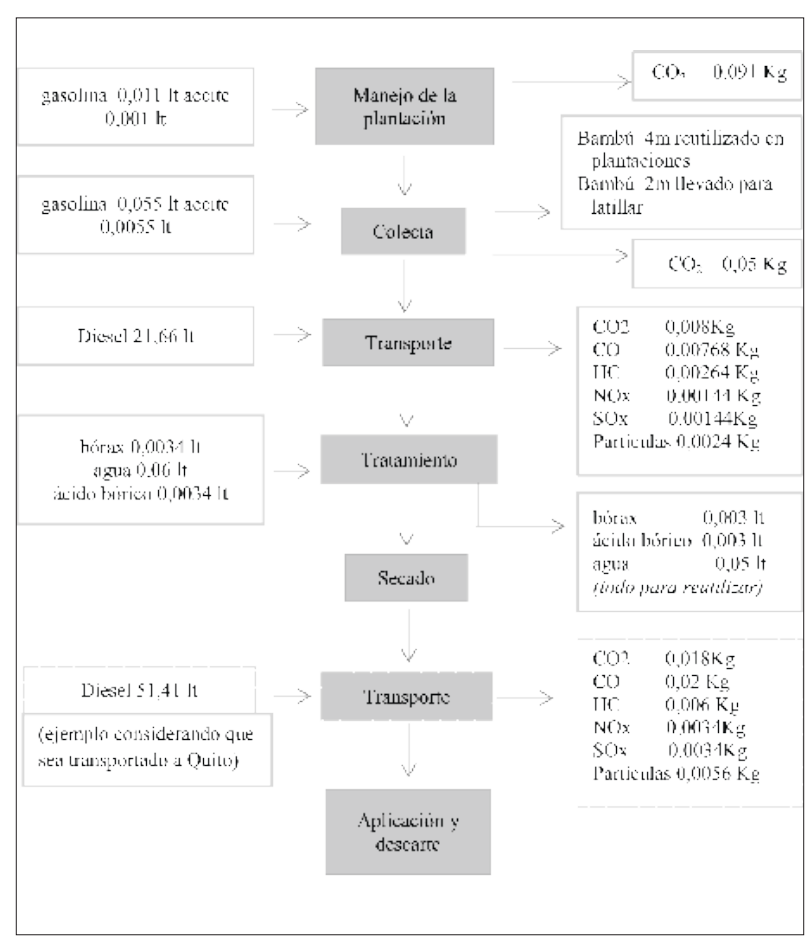

Figura 7. Inventario de la producción de 1 tallo de bambú rollizo tratado de $6 \mathrm{~m}$ de longitud 


\section{CONCLUSIONES Y RECOMENDACIONES}

En el proceso de producción de bambú rollizo tratado la mayoría de las fases se realizan manualmente, se aprovechan las condiciones naturales del medio (y de la planta) para el desarrollo de los cultivos, y se ha tenido una preocupación por la reutilización de residuos. Estos tres factores influyen positivamente en la disminución de impacto ambiental del producto.

Sin embargo, a medida que el proceso se vaya mecanizando, será fundamental mantener la misma preocupación por el manejo de residuos, uno de los puntos críticos es que en mayor escala el impacto de tratamiento químico del material podría repercutir en el entorno próximo (sobre todo en el suelo) y en la salud de las personas que manipulen el preservante.

El factor que más ha elevado la cantidad de emisiones contaminantes durante el proceso estudiado es el transporte del material en camión a diesel. Una opción para atenuar este impacto es aproximar los sitios de tratamiento de bambú a las plantaciones, la otra es buscar medios de transporte alternativos. Este punto también demuestra la importancia de difundir el uso del material en las regiones próximas.

Los datos de este trabajo servirán de base para estudios de ciclos de vida de la amplia gama de subproductos manufacturados con esta especie de bambú en el país, principalmente laminados. Así como para nuevas investigaciones que comparen inventarios de ciclos de vida con otros materiales que tendrían aplicaciones similares.

\section{AGRADECIMIENTOS}

A Eric Briones y a INBAR Latinoamérica (International Network for Bamboo and Ratan) por compartir los datos sobre el Dendrocalamus asper localizado en el noroccidente de Pichincha, indispensables para desarrollar este trabajo.

\section{REFERENCIAS BIBLIOGRÁFICAS}

Alfaro, M. (2010). Estudio de ubicación de lugaresy personas que poseen caña Guadua y bambú gigante en el noroccidente de la provincia de Pichincha. Quito: Gobierno de la Provincia de Pichincha e INBAR.

Braun, S., Gorenstin, L., Schmal, M. ( ) A eliminação catalítica dos particulados de diesel. Recuperado de: http://www.seer. ufrgs.br/index.php/ambienteconstruido/ article/view/3541/1942 (accedido el 07/10/2015)

Hernández, A., Montoya, J., Camargo, J.C. (2012). Análisis del ciclo de vida aplicado a la fase de extracción de guadua en el Eje Cafetero colombiano. Recursos naturales y ambiente 1 (1), 68-76. Recuperado de: http://bibliotecaorton.catie.ac.cr (accedido el 08/10/2015).

International Energy Agency - IEA(2014). CO2 Emissions from fuel combustion. Paris, France. 136p. Recuperado de: http:// www.iea.org/publications (accedido el 07/10/2015).

International Organization for StandardizationISO (2006). Environmental management. Life cycle assessment - Principles and framework. ISO 14040:2006. Geneve, Switzerland.

International Organization for Standardization ISO (1998). Environmental management, Life cycle assessment - Goal and scope definition and inventory analysis. ISO 14041:1998. Geneve, Switzerland.

Lugt, P; Vogtländer, J.; Brezet, H. (2009) Bamboo: a sustainable solution for Western Europe design cases, LCAs and land-use. New Delhi, India, Center for Indian Bamboo Technology. INBAR Technical Report no. 30. Recuperado de: http://www.bambooteam. com/pablo/200810\%20INBAR\%20TR\%20 30\%20v2.7\%20incl\%20figures\%20SMALL. pdf (accedido el 08/10/2015).

Soares, S. R., y Pereira S. W. (2004). Inventário da produção de pisos e tijolos cerâmicos no contexto da análise do ciclo de vida. Ambiente Construido, 4(2), 83-94.

Soares, S. R., De Souza, D. M., Pereira, S. W. (2006). A avaliação do ciclo de vida no contexto da construção civil. En: Coletânea 
Habitare - Construção e Meio Ambiente. Sattler, M.A., Ruttkay, F.O. (Eds.). Porto Alegre: Programa de Tecnologia de Habitação. HABITARE.

Suppen, N., Van Hoof, B. (2005). Conceptos básicos del Análisis de Ciclo de Vida y su aplicación en el Ecodiseño. Centro de análisis de ciclo de vida y diseño sustentable. Cuautitlán Izcalli, México. 42p.

Thompson, R.(2015). Materiais sustentáveis, procesos e produção. Editora Senac São Paulo. São Paúlo, Brasil. 224p. 